\title{
Two nuclear localization signals regulate intracellular localization of the duck enteritis virus UL13 protein
}

\author{
Linjiang Yang, ${ }^{*, \dagger, \ddagger}$ Xixia Hu, ${ }^{*, \dagger, \ddagger}$ Anchun Cheng, ${ }^{*, \dagger, \downarrow, 1}$ Mingshu Wang, ${ }^{*, \dagger, \ddagger}$ Renyong Jia, ${ }^{*, \dagger, \ddagger}$ \\ Qiao Yang, ${ }^{*, \dagger, \dagger}$ Ying Wu, ${ }^{*, \dagger, \dagger}$ Shun Chen, ${ }^{*, \dagger, \ddagger}$ Mafeng Liu, ${ }^{*, \dagger, \dagger}$ Dekang Zhu, ${ }^{*, \dagger, \dagger}$ Xumin Ou, ${ }^{*, \dagger, \dagger}$ \\ XingJian Wen, ${ }^{*, \dagger, \ddagger}$ Sai Mao, ${ }^{*, \dagger, \ddagger}$ Di Sun, ${ }^{*}, \dagger, \ddagger$ Shaqiu Zhang, ${ }^{*}, \dagger, \ddagger$ Xinxin Zhao, ${ }^{*}, \dagger, \ddagger$ Juan Huang, ${ }^{*}, \dagger, \ddagger$ \\ Qun Gao, ${ }^{*, \dagger, \ddagger}$ Yunya Liu, ${ }^{*, \dagger, \ddagger}$ Yanling Yu, ${ }^{*, \dagger, \ddagger}$ Ling Zhang, ${ }^{*, \dagger, \ddagger}$ Bin Tian, ${ }^{*, \ddagger}$ Leichang Pan, ${ }^{*, \ddagger}$ and \\ Xiaoyue Chen ${ }^{*, \dagger, \ddagger}$
}

*Institute of Preventive Veterinary Medicine, Sichuan Agricultural University, Wenjiang, Chengdu City, Sichuan 611130, PR China ${ }^{\dagger}$ Key Laboratory of Animal Disease and Human Health of Sichuan Province, Sichuan Agricultural University, Wenjiang, Chengdu City, Sichuan 611130, PR China; and ${ }^{\ddagger}$ Avian Disease Research Center, College of Veterinary Medicine, Sichuan Agricultural University, Wenjiang, Chengdu City, Sichuan 611130, PR China

\begin{abstract}
Duck enteritis virus (DEV) multifunctional tegument protein $U L 13$ is predicted to be a conserved herpesvirus protein kinase; however, little is known about its subcellular localization signal. In this study, through transfection of 2 predicted nuclear signals of DEV UL13 fused to enhanced green fluorescent protein, 2 bipartite nuclear localization signals (NLS) were identified. We found that ivermectin blocked the NLS-mediated nuclear import of DEV UL13, showing that the nuclear localization signal of DEV UL13 is a classical importin $\alpha$ - and $\beta$-dependent process. We
\end{abstract}

constructed a DEV UL13 mutant strain in which the NLS of DEV UL13 was deleted to explore whether deletion of the NLS affects viral replication. Amino acids 4 to 7 and 90 to 96 were predicted to be NLSs, further proving that nuclear import occurs via a classical importin $\alpha$ - and $\beta$-dependent process. We also found that the NLS of pUL13 had no effect on DEV replication in cell culture. Our study enhances the understanding of DEV pUL13. Taken together, these results provide significant information regarding the biological function of pUL13 during DEV infection.

Key words: duck enteritis virus, conserved herpesvirus protein kinase, UL13, NLS

2021 Poultry Science 100:26-38

https://doi.org/10.1016/j.psj.2020.09.069

\section{INTRODUCTION}

Duck enteritis virus (DEV), a member of the alphaherpesvirinae subfamily, can cause serious clinical symptoms and pathological changes, such as vascular injury, tissue hemorrhage, gastrointestinal mucosal papulosis-like lesions, and degeneration of lymphoid and parenchymal organs (Cheng, 2015). In addition, DEV can lead to acute fever, septicemia, and infectious diseases in ducks, geese, swans, and other birds. The morbidity and mortality of infected or unprotected ducks are very high. This disease often triggers severe economic losses to the global waterfowl industry (Dhama et al., 2017).

(C) 2020 Published by Elsevier Inc. on behalf of Poultry Science Association Inc. This is an open access article under the CC BY-NC-ND license (http://creativecommons.org/licenses/by-nc-nd/4.0/).

Received July 4, 2020.

Accepted September 23, 2020.

${ }^{1}$ Corresponding author: chenganchun@vip.163.com
DEV is a member of the alphaherpesvirinae subfamily of the Herpesviridae family (Cheng, 2015). DEV infection, known as duck plague (DP) or duck viral enteritis (DVE), is among the most widespread and devastating disease in waterfowl (Cheng, 2015). DEV is a linear double-stranded DNA virus that contains 78 open reading frames. The $U L 13$ gene of $\mathrm{DEV}$, which is predicted to encode a serine- or threonine-conserved herpesvirus protein kinase (CHPK), was previously identified by our team (Wu et al., 2012; Hu et al., 2017). There have been reports on DEV pUL13. To study the function of DEV pUL13, it is necessary to know the localization of pUL13 in the cells. The distribution of pUL13 in the nucleus and cytoplasm of DEV-infected cells was observed by indirect immunofluorescence analysis (IFA). Expression of DEV pUL13 fused to green fluorescent protein (GFP) resulted in green fluorescence in the nucleus. Studies have shown that proteins of larger than $40 \mathrm{kDa}$ rely on active transport (Isegawa et al., 2008; Gershburg et al., 2010). Homologous proteins of DEV 
pUL13, such as the UL97 protein of human cytomegalovirus (HCMV), BGLF4 of Epstein-Barr virus (EBV), and U69 of human herpesvirus 6 (HHV-6), have been reported to be localized to the nucleus (Leen et al., 2002; Gershburg et al., 2004), and all these proteins contain nuclear localization signals (NLS) that regulate their nuclear entry (Gershburg et al., 2004). CHPK can autophosphorylate and phosphorylate certain viral and cellular proteins that help the virus replicate and spread, and many of these activities occur in the nucleus. For example, CHPK have been reported to participate in the phosphorylation of nuclear lamina components to facilitate the nuclear egress of progeny capsids (Krosky et al., 2003; Marschall et al., 2005; Prichard et al., 2005; Akihisa et al., 2006; Lee et al., 2008; CanoMonreal et al., 2009). In addition to participating in nuclear egress, CHPK have been proven to influence viral replication and the cell cycle. CHPK affect viral replication by phosphorylating DNA polymerase processivity factors, such as UL44 of HCMV and BMRF1 of EBV (Marschall et al., 2003; Yang et al., 2008), histones (Tarakanova et al., 2007), and RNA polymerase II (Baek et al., 2004; Tamrakar et al., 2005), to promote viral DNA replication and protein expression. In recent years, the EBV BGLF4 protein has been demonstrated to inhibit cell cycle G1 or S progression and induce chromosomal abnormalities (Chang et al., 2012; Paladino et al., 2014). Moreover, herpes simplex virus 1 (HSV-1) pUL13 has been found to stimulate the expression of suppressor of cytokine signaling 3 (SOCS-3), a negative regulator of IFN, to avoid the interferon response. It has also been reported that CHPKs of HCMV, EBV, Kaposi's sarcoma-associated herpesvirus (KSHV), and murine herpesvirus-68 (MHV-68) can subvert the type I IFN response by inhibiting the activity of interferon regulatory factor 3 (IRF-3) (Yokota et al., 2004; Hwang et al., 2009; Wang et al., 2009). Furthermore, some, but not all, CHPKs of betaherpesvirinae and gammaherpesvirinae can phosphorylate the antiviral nucleoside analog ganciclovir (GCV) (Littler et al., 1992; Michel et al., 1998; Leen et al., 2002), which is also present in the nucleus. Amino acid (aa) sequence analysis has revealed that pUL13 shows more than $35 \%$ similarity with reported CHPKs, and investigation of DEV UL13 will provide information about the molecular biological characteristics of DEV and about the CHPK family.

The characteristics of some DEV genes from DEV have been reported by our team (Ma et al., 2018; You et al., 2018; Yang et al., 2019, 2020; Zhao et al., 2019; Zhou et al., 2020). However, to date, there have been few reports on the function of the DEV UL13 protein. Some of the characteristics of DEV pUL13 were identified in our previous study. This report will complement our findings on the localization of DEV UL13 and lay the foundation for studying its function in the nucleus.

\section{MATERIALS AND METHODS}

\section{Cells and Virus}

The previously reported DEV CHv strain (Gen Bank No. JQ647509) was maintained in our laboratory (Zhao et al., 2008; Chang et al., 2009) and slightly changes. Briefly, duck embryo fibroblasts (DEF) were propagated in Minimal Essential Medium (MEM; Gibco-BRL, Grand Island, NY) provided by our laboratory supplemented with $10 \%(\mathrm{v} / \mathrm{v})$ fetal bovine serum (FBS; Gibco-BRL, Grand Island, NY) and incubated at $37^{\circ} \mathrm{C}$ with $5 \% \mathrm{CO}_{2}$.

\section{Analysis of Sequence Motifs Within UL13}

Putative NLS within the UL13 coding sequence were identified using Protein Subcellular Localization Prediction Tool (PSORT) II prediction (https://psort.hgc.jp/ form2.html) (Nakai and Horton, 1999). Sequence analysis using PSORT II predicted 2 potential NLS in the arginine-rich regions of UL13, namely, Arg-Arg-ArgArg (RRRR; aas 4-7) and Pro-Gly-Lys-Arg-Lys-ThrLys (PGKRKTK; aas 90-96) (Supplementary Figure 1).

\section{Construction of Recombinant Plasmids}

Briefly, the full-length UL13 sequence was amplified from DEV chromosomal DNA using F-UL13F and F-UL13R primers designed by Oligo 7. UL13- $\Delta$ NLS1 was PCR amplified from DEV chromosomal DNA using the primers $\Delta$ NLS1F and F-UL13R. UL13- $\Delta$ NLS2 was

Table 1. Oligonucleotide primers used in this work (NCBI number: JQ647509).

\begin{tabular}{ll}
\hline \hline Oligonucleotide primer & \multicolumn{1}{c}{ Sequencea $\left(5^{\prime}-3^{\prime}\right)$} \\
\hline T-UL13 forward primer & GGATCCCTGGTGGCTACGGAGAG \\
T-UL13 reverse primer & AAGCTTCCAAGGGCGTATATGTC \\
F-UL13 forward primer & ccAAGCTTATGGCTGGACGAAGACG \\
F-UL13 reverse primer & ccgGAATTCTGTTATAATCCACAATAGAG \\
$\triangle$ NLS1 forward primer & cccAAGCTTATGGCTGGAAGCCCTATTAGCGAAATG \\
$\triangle$ NLS2 reverse primer & CATCTACTATCCTTTCTCTCTTTACTG \\
$\triangle$ NLS2 forward primer & GAAAGGATAGTAGATGGAGCCTGG \\
GFP forward primer & cccAAGCTTATGGTGAGCAAGGGCG \\
GFP reverse primer & ccgGAATCTGATTATGATCTAGAGTCG \\
NLS1 forward primer & cccAAGCTTATGGCTGGACGAAGACGACGAAGCCCTATGGTGAGCAAGGGCG \\
NLS2 forward primer & ccAAGCTTATGAAGGATCCTGGCAAACGTAAGACAAAGAGTAGAATGGTGAG CAAGGGCG \\
NLS1 and NLS2 forward primer & cccAAGCTTATGGCTGGACGAAGACGACGAATGAAGGATCCTGGCAAAC \\
\hline
\end{tabular}


Table 2. Primers used for Red recombination to construct the CHv-BAC-UL13- $\Delta$ NLS (NCBI number: JQ6475609).

\begin{tabular}{ll}
\hline \hline Oligonucleotide primer & \multicolumn{1}{c}{ Sequence $\left(5^{\prime}-3^{\prime}\right)$} \\
\hline pKD46 forward primer & AAAGCCGCAGAGCAGAAGGTGG \\
pKD46 reverse primer & GGTAAACGGGCATTTCAGTTCAAGG \\
$\Delta$ UL13 and UL14 forward primer & ATATGATTTTTTTTCCTACTCTATTGAATAGTGCGCACT \\
$\Delta$ UL13 and UL14 reverse primer & CTCGCTAACGTGTAGGCTGGAGCTGCTTC \\
UL13- $\Delta$ NLS forward primer 1 & TCGTTTGCAGTGATGTACTGGCGATGAGCTACCATCTATATCCCCACTCA \\
UL13- $\Delta$ NLS forward primer 1X & CTTCACATAATACGCCACTGATC \\
UL13- $\Delta$ NLS reverse primer 1 & GGCGAAAGGCTGCAATACG \\
UL13- $\Delta$ NLS forward primer 2 & CCGATAGGATTCATTTCGCTAATAGGGCTTCCAGCCATTCGTTACCAGATAGTC \\
FRT-Kan forward primer & GACTATCTGGTAACGAATGGCTGGAAGCCCTATTAGCGAAATG AATCCTATCGG \\
FRT-Kan R & AGAAGCGCCGCTCCTCTATTGTGGATTTATAACTAAGTTAGGCTGGAGCTGCTTC \\
& ACGTTTGCAGTGATGTACTGGCGATGAGCTACCATCTATATCCCCACTCATGGT \\
& AGCATATGAATATCCTCCTTAG \\
\hline
\end{tabular}

amplified by overlapping PCR from DEV chromosomal DNA using F-UL13F, $\triangle \mathrm{NLS} 2 \mathrm{R}, \Delta \mathrm{NLS} 2 \mathrm{~F}$, and FUL13R primers. UL13-ANLS1and UL13-ANLS2 were PCR amplified from pEGFP-N1-UL13- $\Delta$ NLS2 DNA using $\Delta \mathrm{NLS1F}$ and F-UL13R primers (Table 1). The PCR fragments were purified and digested with EcoR I and Hind III and ligated into the pEGFP-N1 plasmid digested with the corresponding restriction endonucleases. The ligation mixtures were introduced into $\mathrm{CaCl}_{2}$-competent Escherichia coli $\mathrm{DH} 5 \alpha$ cells, and transformants were selected on Luria-Bertani plates containing $50 \mu \mathrm{g} / \mathrm{mL}$ Kan. The clones were screened by PCR with corresponding primers. The validity of the sequences was determined by sequencing. The GFP, NLS1-GFP, NLS2-GFP, and NLS1\&NLS2-GFP genes were amplified from pEGFP-N1 DNA by PCR using GFPF and GFPR primers; NLS1F and GFPR; NLS2F and GFPR; and NLS1\&NLS2F and GFPR primers, respectively (Table 1 ). These PCR fragments were purified and digested with EcoRI and HindIII. Then, the purified fragments were ligated into the pcDNA3.1(+) plasmid digested with the corresponding restriction endonucleases. The ligation mixtures were introduced into the $\mathrm{CaCl}_{2}$-competent $E$. coli $\mathrm{DH} 5 \alpha$ strain, and transformants were selected on Luria-Bertani plates containing $100 \mu \mathrm{g} / \mathrm{mL}$ Amp. The clones were screened by PCR with the corresponding primers. Sequencing was used for confirmation.

\section{Immunofluorescence Assay}

The experimental protocol was carried out as described previously with slight modifications (Zhang et al., 2010). Briefly, duck embryo fibroblasts (DEF) were fixed with $4 \%$ paraformaldehyde $16 \mathrm{~h}$ after transfection, permeabilized with $0.1 \%$ Triton X-100, and blocked with BSA for $1 \mathrm{~h}$. The cells were incubated with rabbit anti- UL13 antiserum (1:200 dilution) for $1 \mathrm{~h}$ at $37^{\circ} \mathrm{C}$ and then with a fluorescein isothiocyanate (FITC)-conjugated goat antirabbit antibody conjugate diluted at 1:200 (Zhongshan, Beijing, China) for $30 \mathrm{~min}$. The nuclei were stained with DAPI, and the cells were observed using a Nikon ECLIPSE 80i microscope.

\section{Analysis of Pharmaceutical Inhibition of Nucleus Cytoplasm Transport}

The experimental protocol was carried out as described previously (Chang et al., 2010; Shen et al., 2010). Briefly, DEF were cultured in MEM supplemented with $10 \%(\mathrm{v} / \mathrm{v}) \mathrm{FBS}$ at $37^{\circ} \mathrm{C}$ with $5 \% \mathrm{CO}_{2}$. The pEGFP-N1-UL13 or pcDNA3.1(+)-NLS1\&NLS2GFP recombinant plasmid $(2.5 \mu \mathrm{g}$ per well) was transfected into DEF in 6-well cell culture plates with Lipofectamine 2000 (Thermo Fisher, Waltham, MA) according to the manufacturer's instructions. Where appropriate, the cells were treated with ivermectin at

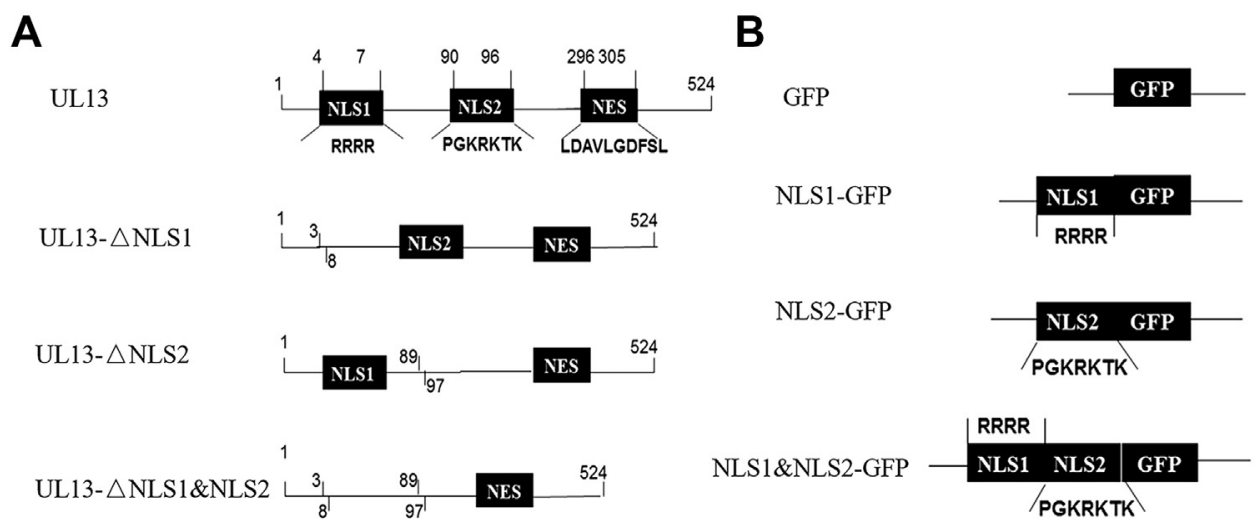

Figure 1. Map of the UL13 recombinant plasmids. (A) Construction of the UL13 NLS deletion plasmids with the EGFP-N1 vector. (B) Construction of the UL13 NLS and EGFP fusion plasmids with the pc-DNA 3.1(+)vector. 
A

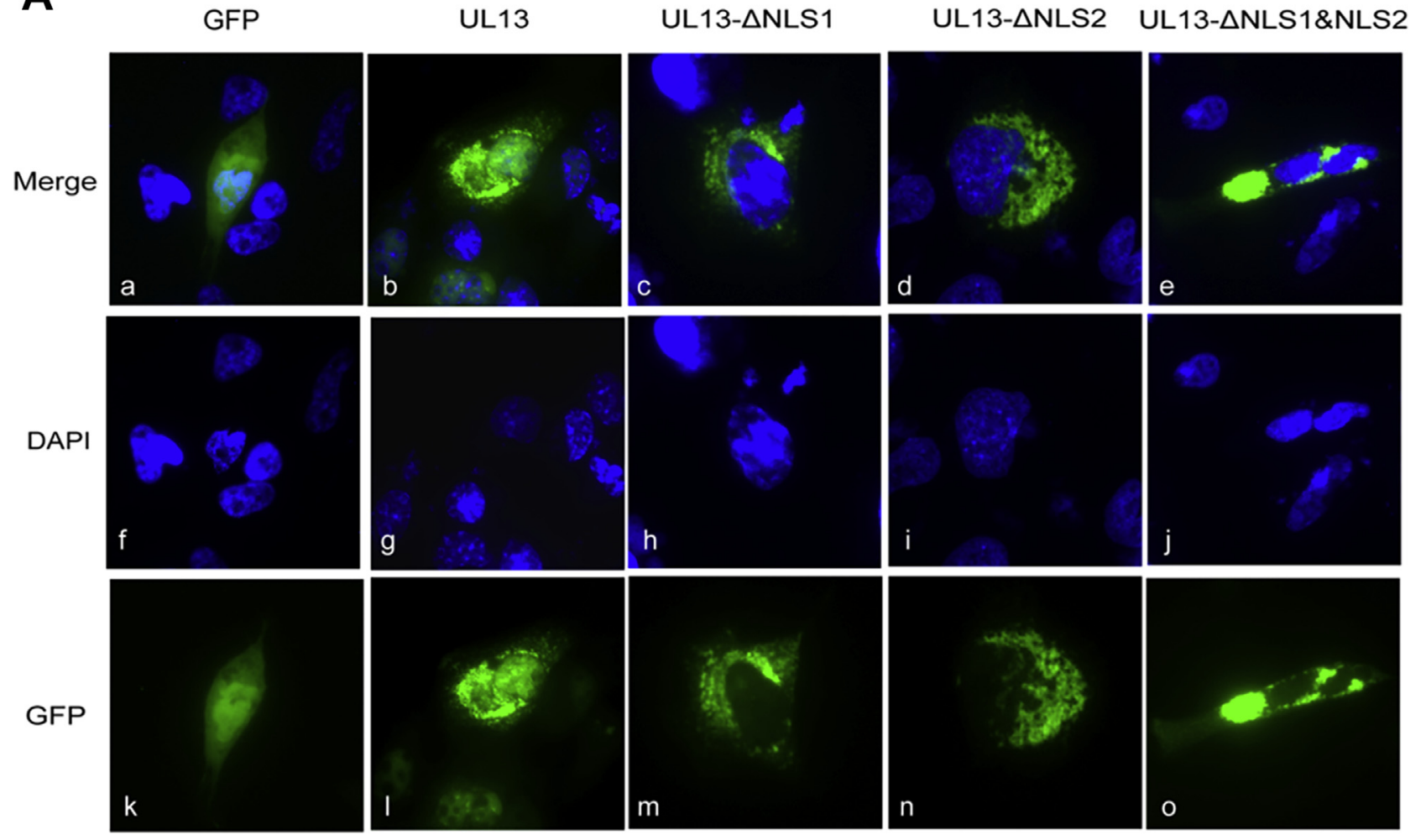

B

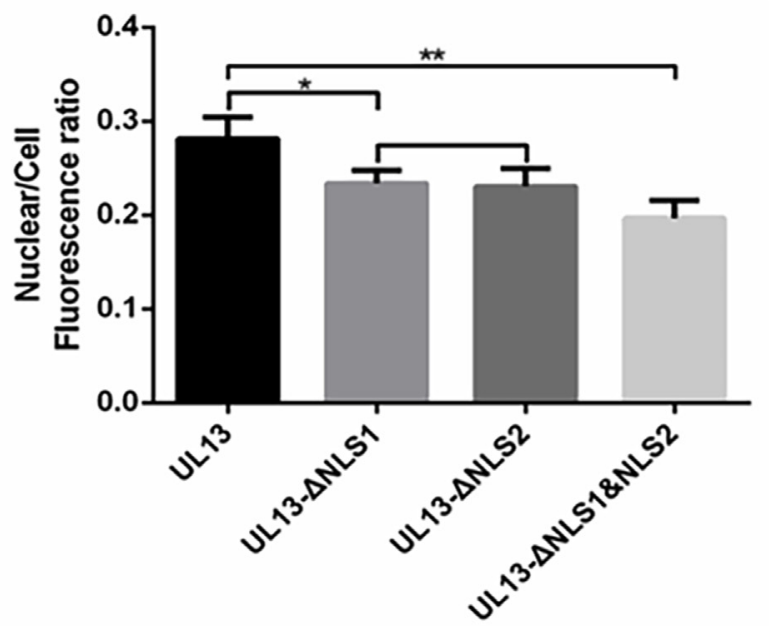

Figure 2. Localization of the UL13 and UL13- $\Delta$ NLS proteins in transfected DEFs. (A) Verification of UL13 and UL13- $\Delta$ NLS fusion protein localization via IFA. The pEGFP-N1- UL13 and UL13- $\Delta$ NLS plasmids were transfected into DEFs. Sixteen hours post transfection, the cells were fixed, permeabilized and incubated with rabbit anti-UL13 antiserum. The nuclei were stained with DAPI (blue). The expression and distribution of GFP protein (green) was monitored by fluorescence microscopy. Images were taken in separate channels using a $40 \times$ objective and merged in SPOT software. (B) Nuclear cytoplasmic fluorescence ratio. The ratio of nuclear and cytoplasmic fluorescence distribution in the recombinant plasmid transfected group was calculated by Image-Pro Plus. Fifty cells from each group were analyzed. The ratio of nuclear and cytoplasmic fluorescence in the pEGFP-UL13- $\Delta$ NLS1, pEGFP-UL13- $\Delta$ NLS2, and pEGFP-UL13- $\Delta$ NLS1 and $\Delta$ NLS2 recombinant plasmid transfected groups was analyzed by GraphPad Prism 6.0 software. ${ }^{*} P<0.05 ; * *<0.01$.

a final concentration of $25 \mu \mathrm{mol} / \mathrm{L}$ for $1 \mathrm{~h}$ before imaging (Nishi et al., 1994; Kudo et al., 1998; Wagstaff et al., 2012). The cells were live-imaged $16 \mathrm{~h}$ after transfection using a Nikon ECLIPSE 80i microscope. To determine the nuclear cytoplasmic fluorescence ratio, digitized images were analyzed using Image-Pro Plus software (Media Cybernetics, Inc., Rockville, MD). Statistical analysis was performed using GraphPad Prism 6.0 software based on $t$ test.

\section{Construction of Recombinant Viruses DEV CHV-UL13 $\triangle N L S$}

To generate the DEV CHv- UL13ANLS virus, with deletion of both NLS1 and NLS2 of UL13 deleted, we first generated DEV CHv- $\Delta$ UL13 \& UL14, a UL13 and UL14 gene-deleted recombinant mutant, based on the overlap of UL13 and UL14 gene regions. The target segment (UL13 left arm-FRT-Kan-FRT-UL14 right arm) was 
A
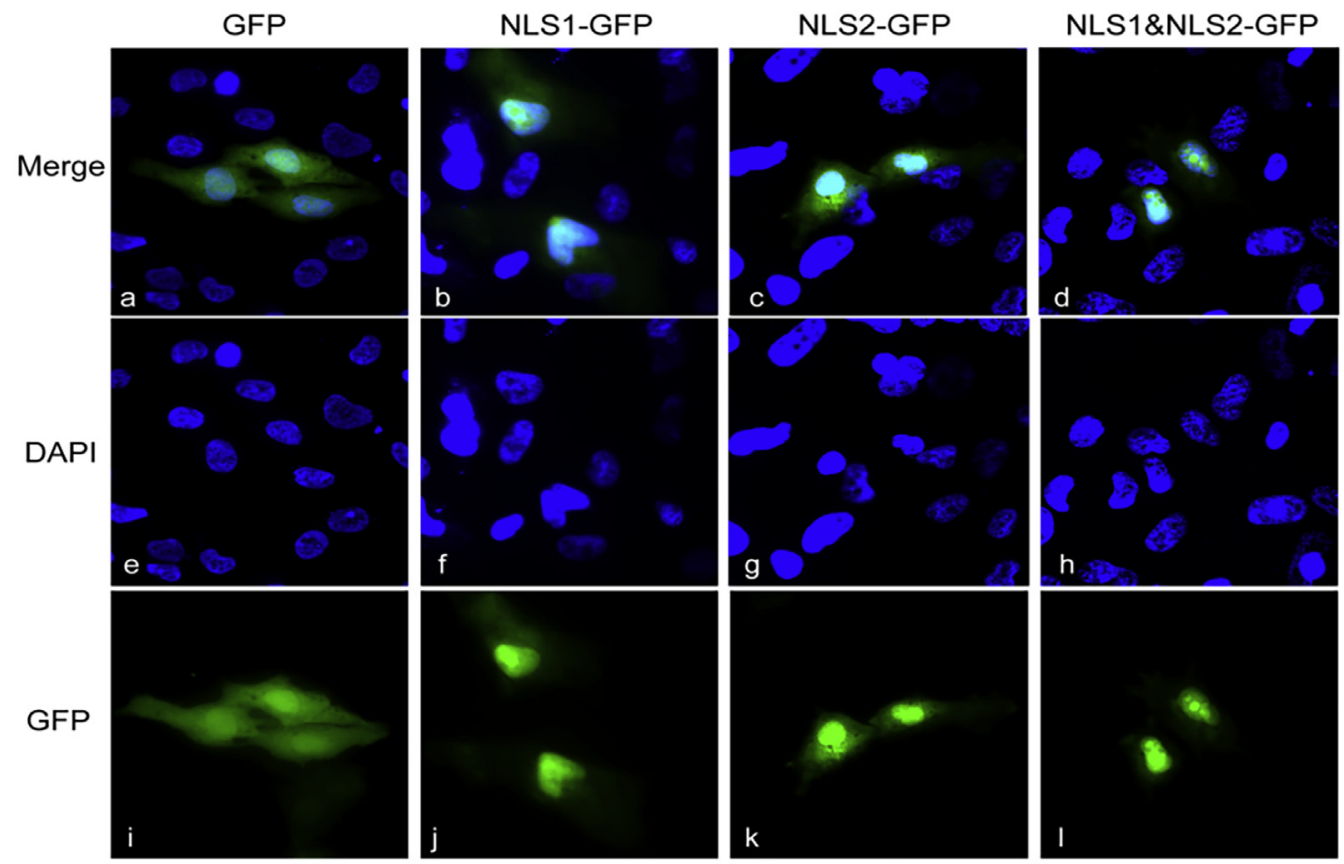

B

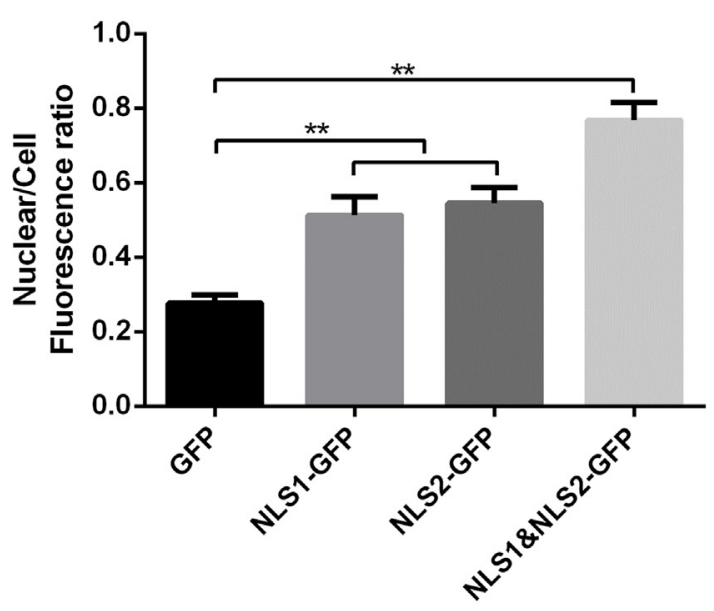

Figure 3. Construction of NLS-GFP recombinant plasmids and localization analysis of the fusion proteins. (A) Localization of the NLS-GFP fusion proteins in transfected DEFs. NLS-GFP recombinants were transfected into DEF cells, which were then fixed $16 \mathrm{~h}$ posttransfection. Images of the GFP reporter (green) and nuclei stained with DAPI (blue) were taken in separate channels using a $40 \times$ objective and merged in SPOT software. (B) Comparison of the nuclear and cytoplasmic fluorescence ratios of GFP and the NLS-GFP fusion protein. The mean nuclear and cytoplasmic fluorescence ratios were quantified using Image-Pro Plus software; differences in the nuclear and cytoplasmic fluorescence ratios of GFP and the NLS1-GFP and NLS2-GFP, NLS1\&NLS2-GFP proteins was analyzed using ANOVA with GraphPad Prism 6.0 software. N = 50 for each group.

PCR-amplified using $\triangle U L 13 \quad \& \quad U L 14 \quad \mathrm{~F}$ and $\triangle U L 138 U L 14 \mathrm{R}$ primers (Table 2). Then, the infectious clone DEV CHv- $\triangle U L 13 \& U L 14-\mathrm{G}$ was generated by using a recombination system based on the genetic manipulation of the DEV CHv-G infectious clone (Ma et al., 2018; You et al., 2018). Briefly, the pKD46 plasmid, which encodes the recombination genes exo, beta, and gam under tight control of the ParaB promoter, was first introduced into E. coli $\mathrm{DH} 10 \mathrm{~B}$ containing the $\mathrm{DEV}$ CHv-G plasmid by electrophoretic transfer. In this system, the UL13\&UL14 coding sequences were replaced with the target segments amplified from the pKD4 plasmid. Then, the pCP20 plasmid was transferred into the aforementioned cells, which contained DEV CHv$\Delta U L 13 \& U L 14^{-} \mathrm{G}$ and a kanamycin-resistant gene cassette. Removal of the kanamycin-resistant cassette was accomplished by incubation at $30^{\circ} \mathrm{C}$ for $8 \mathrm{~h}$ and then at $42^{\circ} \mathrm{C}$ overnight. Then, we generated DEV CHvUL13ANLS-G and the revertant DEV CHv-UL13ANLS R-G based on the same method described previously but with 2 different target segments. The target segment of DEV CHv-UL13ANLS-G, UL13ANLS-UL14-FRT-KanFRT-UL14 right arm was fusion PCR-amplified using

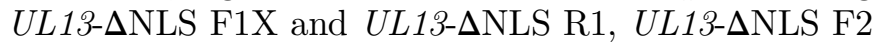
and FRT-Kan $\mathrm{F}$ and $\mathrm{R}$ primers (Table 2) with the pEGFP-N1- UL13- NLS1\& $\Delta$ NLS2 plasmids, the CHv 

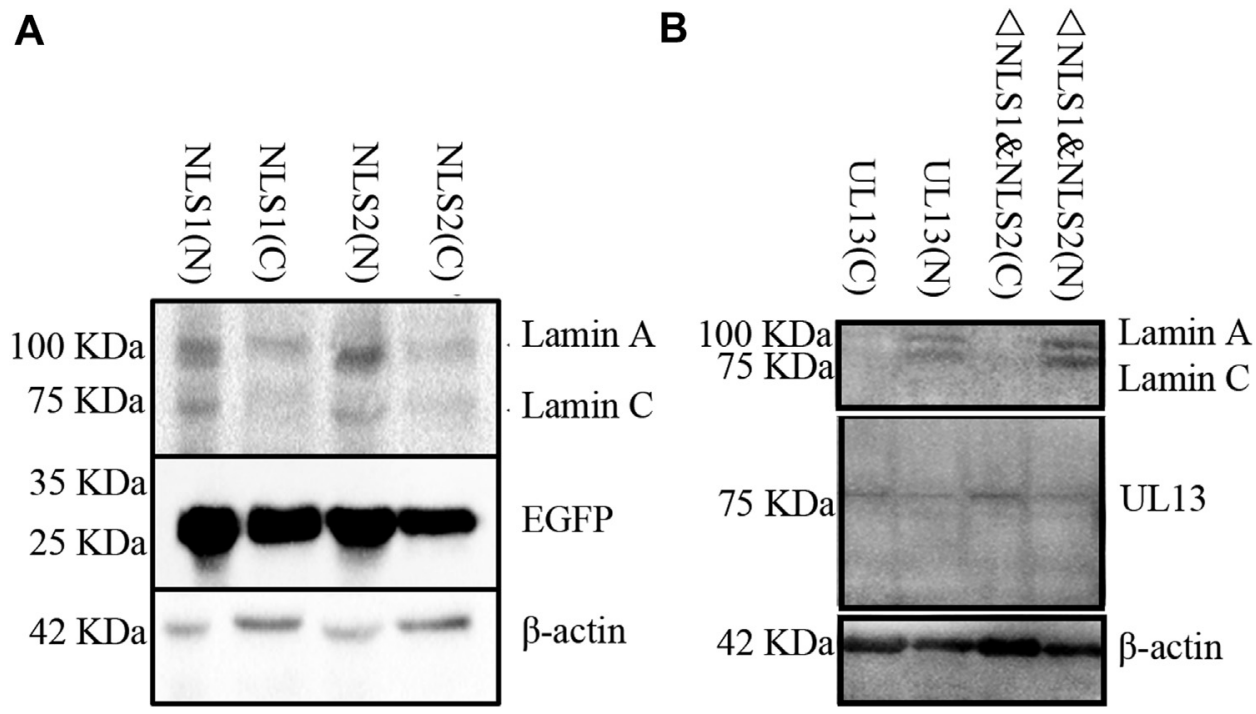

Figure 4. Western blot analysis of the UL13 NLS. (A) The nuclear and cytoplasmic distribution of pUL13-NLS1 and pUL13-NLS2 was analyzed by western blotting. DEFs were transfected with the NLS1 and NLS2 plasmids, and after $48 \mathrm{~h}$, the cells were harvested. (B) The nuclear and cytoplasmic distribution of the pUL13-NLS1\& NLS2- deleted plasmids were analyzed b through western blot. DEF were transfected with the NLS1 and NLS2 plasmids, and after $48 \mathrm{~h}$, the cells were harvested.

genome and the pKD4 plasmid, respectively, as a template; the target segment of DEV CHv- UL13ANLS R-G, UL13-UL14-FRT-Kan-FRT-UL14 right arm was fusion PCR-amplified using the UL13- $\Delta$ NLS F1 and FRT-Kan $\mathrm{F}$ and $\mathrm{R}$ primers (Table 2) with the $\mathrm{CHv}$ genome and the pKD4 plasmid, respectively, as a template. The DEV CHv- UL13ANLS-G and DEV CHv-UL13ANLS R$\mathrm{G}$ infectious clones were identified by sequencing and restriction fragment length polymorphism analysis. Finally, the DEV CHv-UL13ANLS and DEV CHv-UL13ANLS R recombinant viruses were rescued; freshly prepared DEV CHv-UL13ANLS -G and DEV CHv-UL13ANLS R-G plasmids were transfected into DEFs for 1 to $10 \mathrm{~d}$. The cells were examined by fluorescence microscopy until green fluorescence protein seemed sufficiently expressed, at which point, they were harvested after 3 freeze-thaw cycles. After the DEV CHv-UL13ANLS and DEV CHvUL13ANLS R viruses were amplified, viral PCR identification and sequencing of the target region were performed.

\section{Plaque Assay}

The experimental protocol was carried out as described previously (Guo et al., 2009). The cryopreserved recombinant viruses DEV CHv, CHv-UL13 $\Delta$ NLS, and CHv-UL13 $\Delta$ NLS R were diluted with MEM at a 10-fold dilution. Then, a 1:1 mixture of $2 \times \mathrm{MEM}$ and $0.5 \%$ methylcellulose (9004-67-5; J\&K Scientific Ltd., Beijing, China) was added to the plates after the unabsorbed particles were discarded. After incubation at $37^{\circ} \mathrm{C}$ for $6 \mathrm{~d}$, the cells were fixed with $4 \%$ paraformaldehyde and stained with $0.05 \%$ crystal violet (C3886; Haoran Bio, Beijing, China). A total of 50 plaques were randomly chosen for each virus, all plaques were scanned, and plaque diameters were determined in pixels using Adobe Photoshop.

\section{Growth Curve Assay}

The experimental protocol was carried out as described previously with slight modifications (Liu et al., 2016). Briefly, DEFs were infected with DEV CHv, DEV CHv-UL13ANLS, or DEV CHvUL13ANLS R at 200 TCID $_{50}$. The cells were maintained in MEM supplemented with $2 \%$ FBS, and cell and supernatant samples were separately harvested after 6, 12, 24, 48,72 , and $96 \mathrm{~h}$ and used for the growth curve assay after 3 freeze-thaw cycles. A growth curve was produced by measuring the $\mathrm{TCID}_{50}$, which was calculated by the Reed Muench method. Samples collected at different time points were analyzed in triplicate.

Western Blotting As previously described (Yang et al., 2020), DEV-infected DEF cells in 6-well dishes were collected at $7,24,36,48,60$, and $72 \mathrm{~h}$ after infection (hpi), removing the supernatant. After several washes with PBST to remove unbound antibodies, the signal was detected using Western blot Chemiluminescence HRP Substrate (Takara, Dalian, China) according to the manufacturer's instructions.

\section{Nuclear and Cytoplasmic Separation Experiment}

We used the NE-PER Nuclear and Cytoplasmic Extraction Reagents kit to separate nuclei and cytoplasm (78833; Thermo). The protocol was performed in accordance with the instructions. For adherent cells, harvest with trypsin-EDTA and then centrifuge at $500 \times g$ for 5 min. Wash cells by suspending the cell pellet with PBS. Transfer $1-10 \times 10^{6}$ cells to a $1.5-\mathrm{mL}$ microcentrifuge tube and pellet by centrifugation at $500 \times g$ for 2 to $3 \mathrm{~min}$. Use a pipette to carefully remove and discard the supernatant, leaving the cell pellet as dry as possible. Add ice-cold CER I to the cell pellet. Vortex the tube 
A

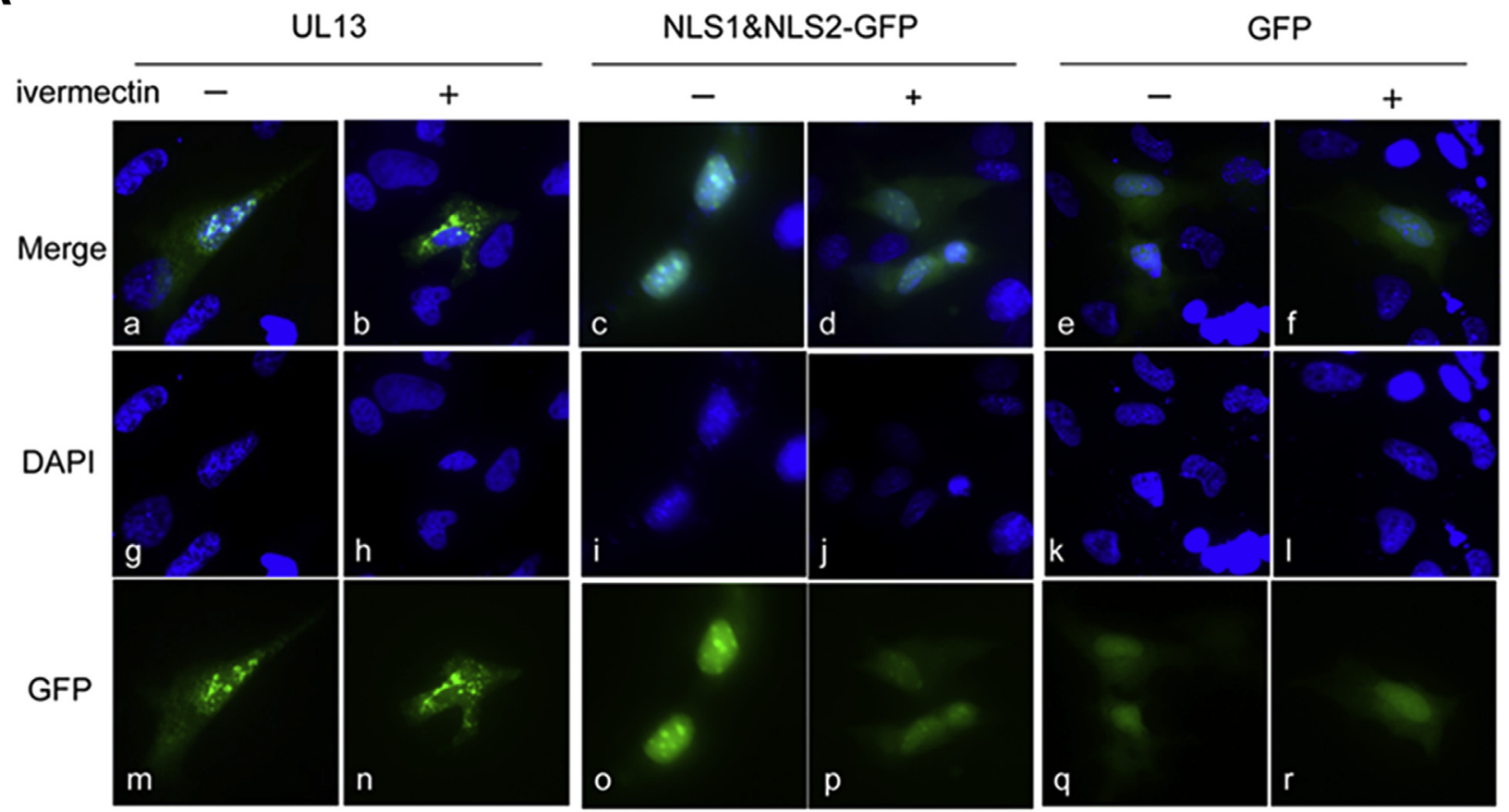

B

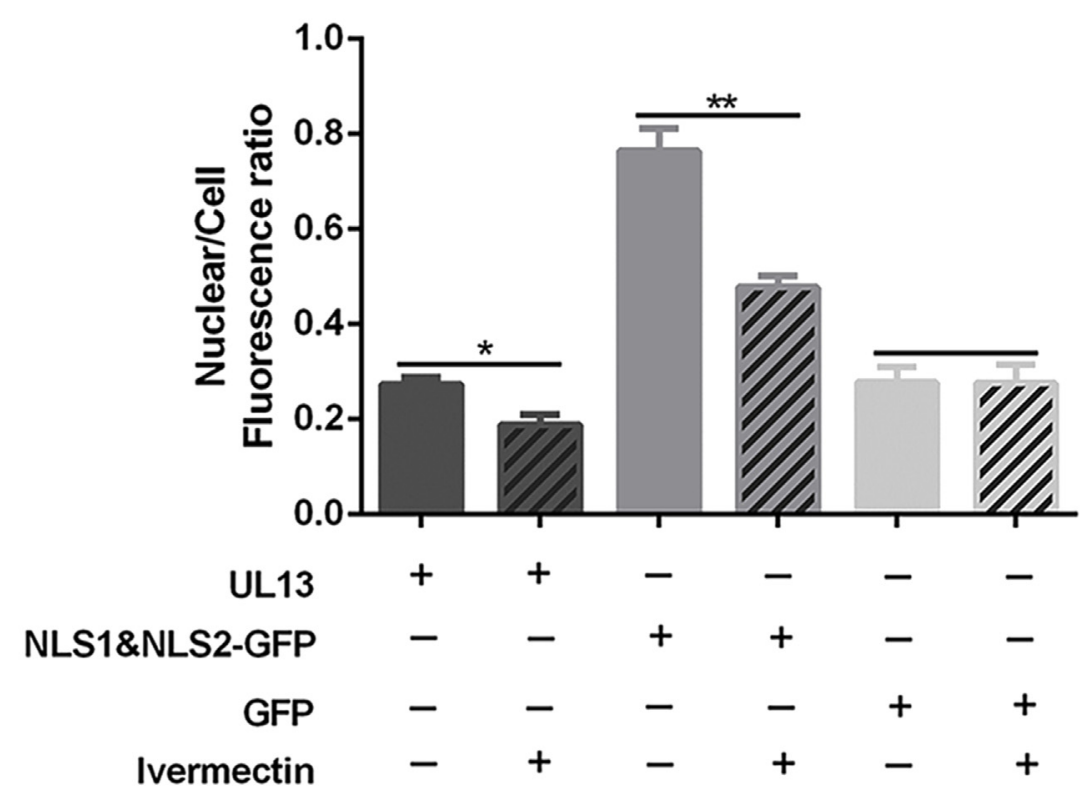

Figure 5. Effect of nuclear import inhibitors on the localization of the DEV pUL13. (A) Localization of the UL13-GFP and NLS1\&NLS2-GFP proteins in cells treated with or without ivermectin. Sixteen hours posttransfection, $25 \mu \mathrm{mol} / \mathrm{L}$ ivermectin was added $1 \mathrm{~h}$ before fixation. Images of the GFP reporter (green) and nuclei stained with DAPI (blue) were taken in separate channels using a $40 \times$ objective and merged in SPOT software. (B) Comparison of the nuclear and cytoplasmic fluorescence ratios of proteins in cells treated with or without ivermectin. The mean nuclear and cytoplasmic fluorescence ratios were quantified using Image-Pro Plus software; the differences in the nuclear and cytoplasmic fluorescence ratios of GFP-NLS1and NLS2-GFP protein in cells treated with or without ivermectin were analyzed using ANOVA with GraphPad Prism 6.0 software. $\mathrm{N}=50$ for each group.

vigorously on the highest setting for $15 \mathrm{~s}$ to fully suspend the cell pellet. Incubate the tube on ice for $10 \mathrm{~min}$. Then, add ice-cold CER II to the tube. Vortex the tube for $5 \mathrm{~s}$ on the highest setting. Incubate tube on ice for $1 \mathrm{~min}$. Vortex the tube for $5 \mathrm{~s}$ on the highest setting. Centrifuge the tube for $5 \mathrm{~min}$ at maximum speed in a microcentrifuge $(\sim 16,000 \times g)$. Immediately transfer the supernatant (cytoplasmic extract) to a clean prechilled tube. Suspend the insoluble (pellet) fraction, which contains nuclei, in ice-cold NER. Vortex on the highest setting for $15 \mathrm{~s}$. Place the sample on ice and continue vortexing for $15 \mathrm{~s}$ every $10 \mathrm{~min}$, for a total of $40 \mathrm{~min}$. Centrifuge the tube at maximum speed $(\sim 16,000 \times g)$ in a microcentrifuge for $10 \mathrm{~min}$. Immediately transfer the supernatant (nuclear 
A

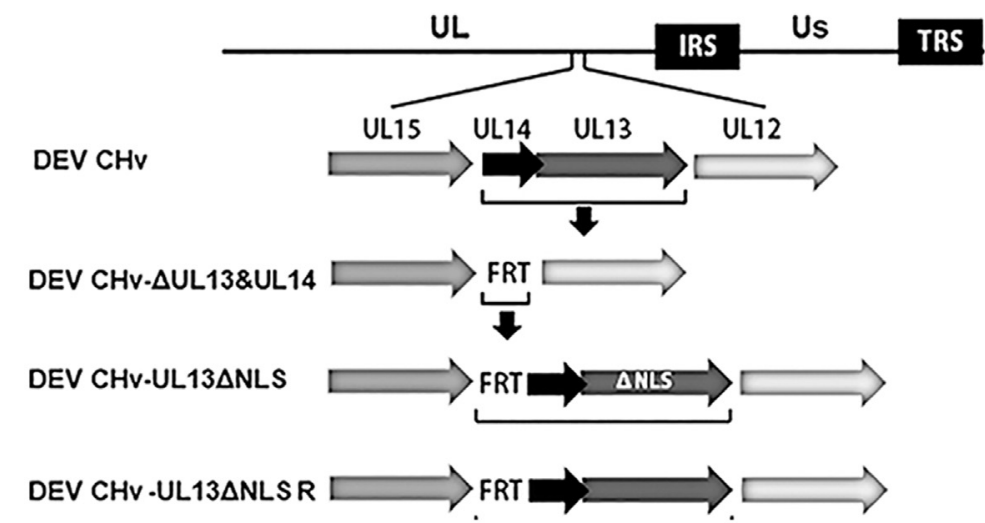

B

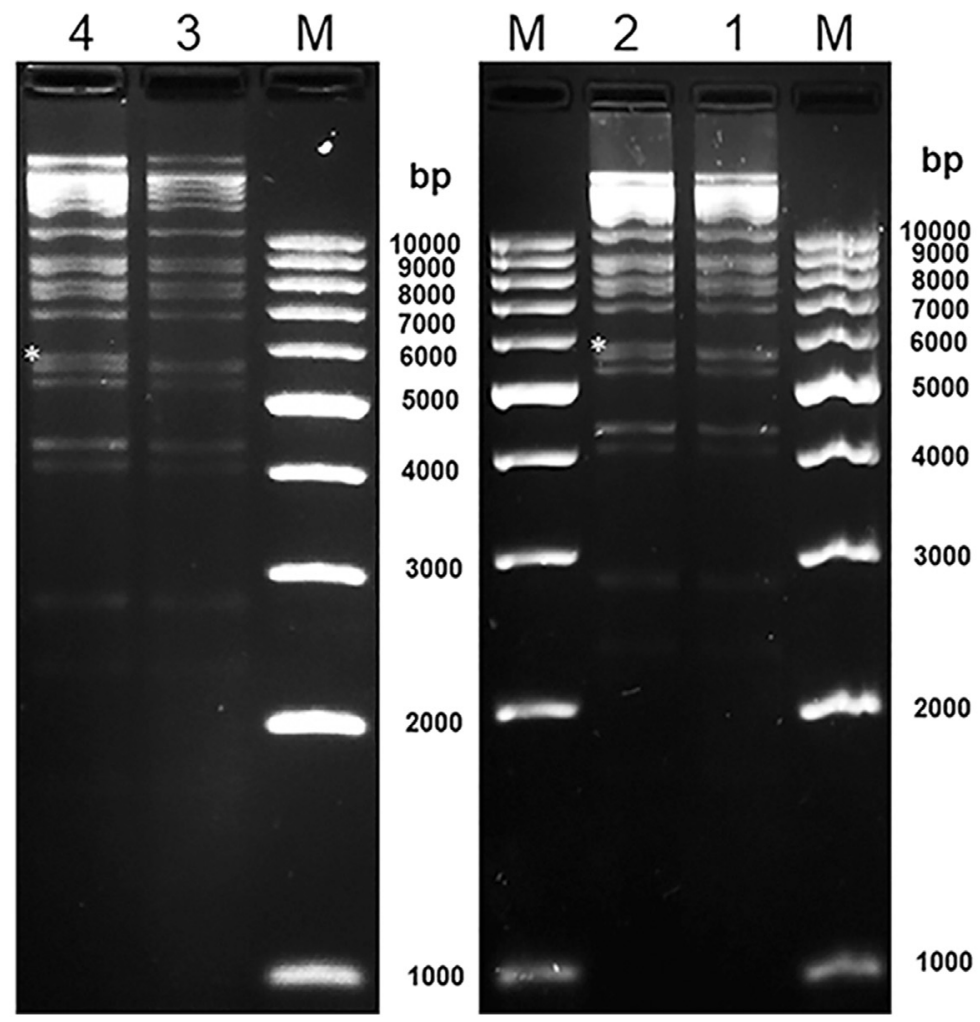

Figure 6. Construction and identification of DEV CHv-UL13 $\Delta$ NLS and DEV CHv-UL13 $\Delta$ NLS R recombinant viruses. (A) Schematic represen-

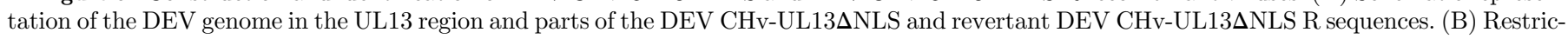
tion Fragment Length Polymorphism analysis. Lane 1 and lane 3, DEV CHv-G plasmids digested with SalI restriction endonuclease; lane 2, DEV

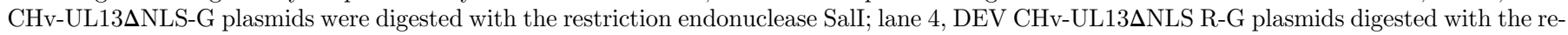
striction endonuclease SalI. Digestion of the deletion and revertant products resulted in shifts of the 5,714-bp fragment of the parental virus (lane 1 and lane 3) to 5,765 bp and 5,798 bp (lane 2 and lane 4), respectively. No extraneous alterations were evident in either clone. M, $1 \mathrm{~kb}$ plus DNA ladder. *different bands.

extract) fraction to a clean prechilled tube. Place on ice. The obtained cytoplasmic nuclear proteins were subjected to Western blotting. Proteins that were resolved by $12 \%$ SDS-PAGE were further transferred to polyvinylidene fluoride (PVDF) membranes. Membranes were blocked for $2 \mathrm{~h}$ in $5 \%$ skim milk, then incubated with primary antibody and probed with HRP-conjugated secondary antibody for $1 \mathrm{~h}$ at $37^{\circ} \mathrm{C}$. All antibodies were diluted in $1 \%$ skim milk. The rabbit anti-UL13 antibody (Hu et al., 2017), rabbit anti- $\beta$-actin antibody (BOSTER, BA2305, CA), and rabbit anti-Lamin A and C antibody
(Signalway Antibody, 41099, MD) were used as primary antibodies. Lamin $\mathrm{A}$ and $\mathrm{C}$ was used as nuclear reference gene. $\beta$-actin was used as cytoplasm reference gene. The secondary antibodies are corresponding goat antirabbit IgG $(\mathrm{H}+\mathrm{L})$-HRP conjugate (Bio-rad, 1706515, CA) and goat antimouse IgG $(\mathrm{H}+\mathrm{L})$-HRP conjugate (Biorad, 1706516, CA). Protein bands were visualized by using ECL Western blotting detection reagents (BioRad, CA) by ChemiDoc MP (Bio-Rad, CA). To determine the nuclear cytoplasmic ratio, protein bands were quantified using Image-Pro Plus software. Statistical 
A

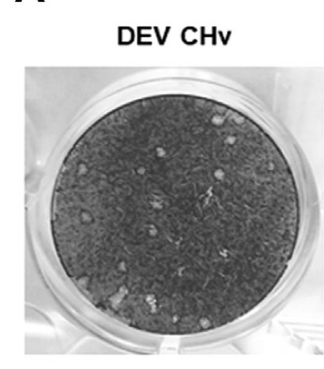

DEV CHV-UL13 $\triangle$ NLS
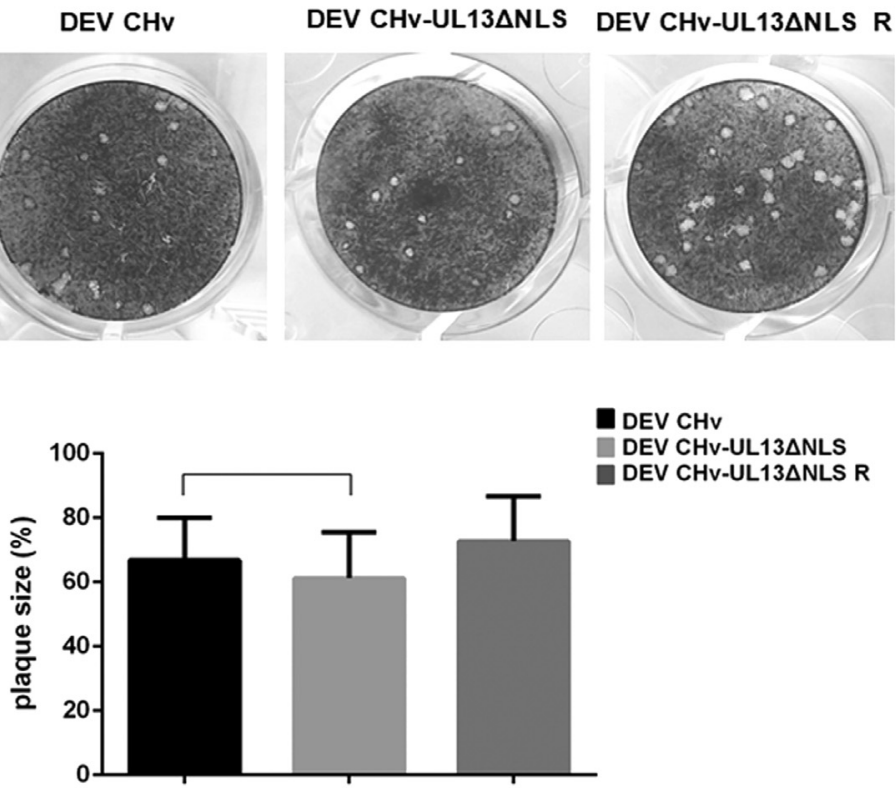

B
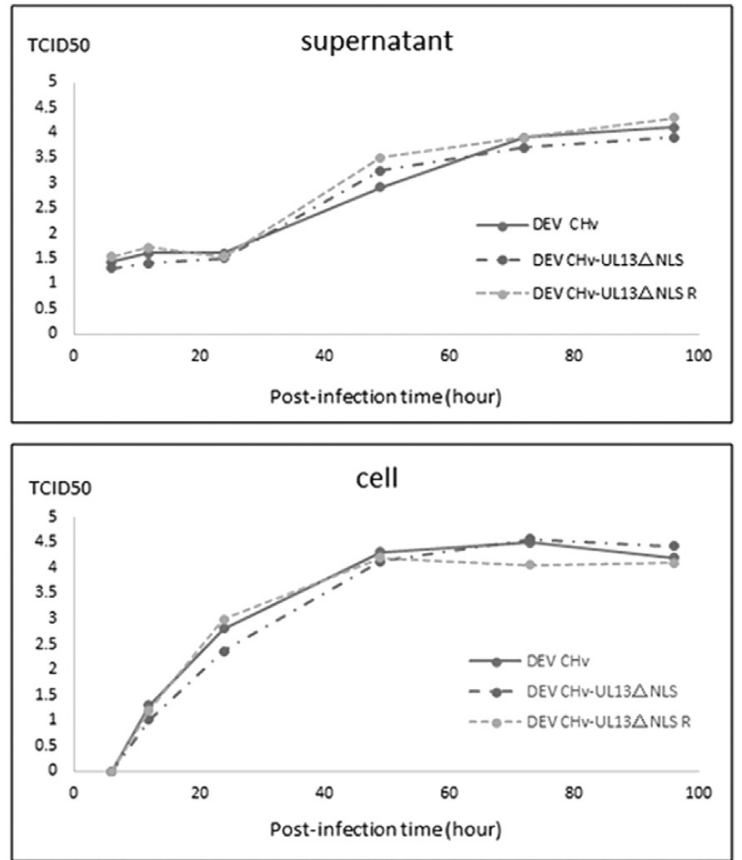

Figure 7. Analysis of the characteristics of the UL13-deletion mutant virus in cultured cells. (A) Plaque assay. The figures represent the phenotypes of the plaques formed by infection with DEV CHv, DEV CHv-UL13ANLS, and DEV CHv-UL13 NLS R. The histogram shows the analysis of the mean are of viral plaques formed by the various viruses following infection. The digital images were analyzed using Adobe Photoshop, and statistical analyses were performed using GraphPad Prism 6.0 software. N = 50 for each group. (B) Growth curve. DEFs were infected with DEV CHv, DEV

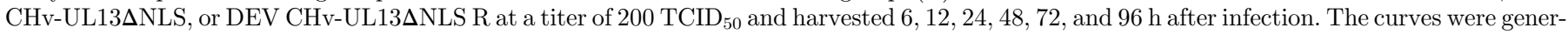
ated based on the viral titers in cells harvested at different time points by determining the TCID 50 using Excel. Significant difference between DEV $\mathrm{CHv}$ - and DEV CHv- $\Delta$ UL13-infected cells were analyzed using GraphPad Prism 6.0 software.

analysis was performed using GraphPad Prism 6.0 software based on $t$ test.

\section{RESULTS}

\section{Localization of Recombinant UL13 and UL13-4NLS Mutant Protein in Transfected DEFs}

We previously determined that DEV pUL13 can enter the nucleus. PSORT II prediction identified aas 4 to 7 (RRRR) and 90 to 96 (PGKRKTK), as putative NLSs (Supplementary Figure 1). To determine the function of these predicted NLSs, we constructed expression plasmids for UL13-GFP fusion proteins and proteins with NLS1, NLS2, or both deleted (Figure 1A) or retained (Figure 1B). After transfection, unlike the fluorescence of the UL13-GFP fusion proteins, which was localized in the nucleus and cytoplasm (Figure 2A, b, g, and l), the majority of fluorescence displayed a cytoplasmic distribution in DEFs when NLS1 or NLS2 was deleted (Figure 1B, c, h, m, d, i, and $\mathrm{n}$ ). Furthermore, the UL13-GFP fusion protein with deletion of both NLS1 and NLS2 was located entirely in the cytoplasm (Figure 2A, e, j, and o). We also analyzed the nuclear compartmentalization of UL13- $\Delta$ NLS. As shown in Figure 2B, compared to the nuclear cytoplasmic fluorescence ratio of $U L 13$, the nuclear fluorescence ratios of $U L 13-\Delta \mathrm{NLS1} \& \mathrm{NLS} 2(P<$
$0.01)$ and the UL13- $\Delta$ NLS1 and UL13- $\Delta$ NLS2 proteins were significantly decreased $(P<0.001$ for both). The decrease in the nuclear cytoplasmic fluorescence ratio was higher for UL13- $N$ NS1\&NLS2 than that for UL13- $\Delta$ NLS1 and UL13- - NLS2. These results suggest that both NLS1 and NLS2 are responsible for and work together to enhance the nuclear localization of DEV pUL13.

\section{Localization of the NLS-GFP Fusion Protein in Transfected DEFs}

To further examine whether NLS1 and NLS2 can import heterologous proteins into the nucleus, sequences encoding NLS1 and NLS2 were fused to GFP to obtain NLS1-GFP, NLS2-GFP, and NLS1\&NLS2-GFP recombinant plasmids, and these plasmids were transfected into DEFs (Figure 1B). Fluorescence assays showed that a greater proportion of the NLS1-GFP and NLS2-GFP fusion proteins was localized in the nucleus than in the cytoplasm (Figure 3A, b, f, j, c, g, and k). In contrast, unlike GFP alone (Figure 3A, a, e, and i), NLS1\&NLS2GFP showed a predominantly nuclear distribution (Figure 3A, d, h, and l). We also analyzed differences in the nuclear and cytoplasmic fluorescence ratios of GFP and NLS-GFP and found that the nuclear cytoplasmic fluorescence ratios of NLS1-GFP, NLS2-GFP, and especially NLS1\&NLS2-GFP were 
significantly higher than the nuclear cytoplasmic fluorescence ratio of GFP $(P<0.001)$ (Figure 3B).

\section{Western Blot Analysis of DEV UL13 NLS1 and NLS2}

We also separated cytoplasmic and nuclear proteins from DEFs and analyzed the quantities of pUL13 distributed in the cytoplasm and nucleus via Western blotting (Figure 4A). Distribution of UL13- $\Delta$ NLS1 and $\Delta$ NLS2 group was less widely distributed than DEV pUL13. We used $\beta$-actin and Lamin A and $\mathrm{C}$ as controls for Western blot analysis of the cytoplasmic nuclear fractions and found that the results were consistent with the IFA results. We also separated cytoplasmic and nuclear proteins from DEF cells expressing the NLS1-GFP or NLS2-GFP plasmid and analyzed the quantities of UL13 protein in the cytoplasm and nuclei via Western blotting (Figure 4B). The expression of pUL13 in the cytoplasm was significantly lower than that in the nucleus in the NLS1-GFP and NLS2-GFP groups. Our results confirmed that both NLS1 and NLS2 of DEV pUL13 act as NLS. Each can mediate protein entry into the nucleus, and the combination of these NLS increases nuclear localization.

\section{Effect of Nuclear Import Inhibitors on the Localization of DEV UL13}

NLS1 and NLS2 of DEV UL13 are predicted to be 4- and 7-aa prototypical monopartite NLS, respectively. Therefore, we tested whether the nuclear import of DEV pUL13 requires an interaction between importin $\alpha$ and $\beta$ by treating UL13 recombinant plasmid transfected DEFs with or without ivermectin. As shown in Figure 5A, the level of DEV pUL13 in the nucleus was lower in the ivermectin group than that in the untreated group at $16 \mathrm{~h}$ after transfection (Figure $5 \mathrm{~A}, \mathrm{a}, \mathrm{g}, \mathrm{m}, \mathrm{b}, \mathrm{h}$, and $\mathrm{n}$ ). The distribution of NLS1\&NLS2-GFP in the nucleus in the ivermectin-treated group was also lower than that in the untreated group (Figure 5A, c, i, o, d, j, and $\mathrm{p})$, whereas there was no change in the distribution of GFP between the ivermectin-treated and untreated groups (Figure 5A, e, k, q, f, l, and r). We also analyzed the differences in the nuclear and cytoplasmic fluorescence ratios of NLS1\&NLS2-GFP and UL13-GFP in $\mathrm{DEF}$ treated with or without ivermectin and found that the ratio of UL13-GFP was significantly lower in the ivermectin-treated group than that in the untreated group $(P<0.001)$, with the mean ratio in the NLS1\&NLS2-GFP groups being reduced from 0.8 to 0.4 after ivermectin treatment. Statistical analysis showed that the nuclear accumulation of NLS1\&NLS2GFP and UL13-GFP was significantly impaired by ivermectin (Figure 5B) $(P<0.01)$. These results suggest that the nuclear import of DEV pUL13 occurs through the classical transport process, which requires an interaction between importin $\alpha$ and $\beta$ and is mediated by the NLS of DEV pUL13.

\section{Nuclear Entry of pUL13 Has No Effect on DEV Replication in Cell Culture}

To examine the effect of the pUL13 nuclear entry on viral proliferation, we constructed $\mathrm{DEV} \mathrm{CHv}^{-}$ UL13ANLS, a mutant in which both NLS1 and NLS2 of DEV UL13 are deleted, and DEV CHv-UL13ANLS $\mathrm{R}$ (a revertant) (Figure 6A). Through Restriction Fragment Length Polymorphism analysis and sequencing, we determined that the DEV CHv-UL13ANLS and DEV CHv-UL13ANLS R recombinant viruses were mutated only at the appropriate target sites (Figure 6B).

A plaque assay indicated that DEV CHv-UL13ANLSinfected DEF grew and that the difference in plaque size between DEV CHv-UL13 NLS-infected DEF, and DEV CHv-infected DEF was not significant (Figure 7A). There was a small difference in the ratios of DEV CHv- UL13ANLS and DEV CHv in both the supernatant and cytoplasm during infection (Figure 7B). The growth curve of DEV CHv-UL13ANLS-infected DEF exhibited the same trend as that of DEV CHv-infected DEF in both the supernatant and cytoplasm during infection (Figure 7C). The phenotype of DEV CHv- $U L 13 \mathrm{R}-$ infected DEF exhibited similar properties.

\section{DISCUSSION}

All herpesviruses encode serine or threonine kinases, which are involved in viral proliferation and organism immunity (Thary et al., 2011). Two types of conserved herpesvirus serine or threonine kinases have been described in the family Herpesviridae: one is conserved only in the alphaherpesvirus subfamily and includes the US3 kinase of HSV; the others are CHPKs, which are conserved across the entire family. Studies on CHPK, especially those of human herpesviruses, have mainly focused on their functions and molecular characteristics, whereas studies on CHPK of animal herpesviruses, such as DEV, are comparatively scarce. In a previous study, our group isolated DEV from the intestine of a sick duck and sequenced the genome (Wu et al., 2012). This analysis showed that DEV encodes a $\mathrm{p} U L 13$ showing $35 \sim 40 \%$ similarity with reported CHPKs of human herpesviruses and 40 50\% similarity with the CHPK of poultry herpesviruses.

Early reports showed that pUL13 can be extracted from the nuclei of HSV-1- and HSV-2-infected cells (Daikoku et al., 1997). The nuclear pore complex allows soluble small molecules to be imported or exported through the nuclear membrane. The transported substances include RNA, ribosomes, proteins, carbohydrates, and signaling molecules (Kabachinski and Schwartz, 2015). Small molecules can pass through the 
nuclear pore complex through diffusion, while large molecules may enter and exit the nucleus with the help of nuclear pore proteins after specific signal sequences are recognized (Miller et al., 1991; Schmidt-Zachmann and Nigg, 1993). NLS sequence are amino acid sequences on proteins that allows their transport into the nucleus. Therefore, proteins with NLS can effectively enter the nucleus through the nuclear pore complex (Ohshima et al., 2012). In conclusion, we speculate that DEV pUL13 also has an NLS.

Based on biological analysis of DEV UL13, 2 basic aaenriched peptides, namely, aas 4 to 7 and aas 90 to 96 , were predicted as potential NLS. This prediction was consistent with the subsequent results, further confirming that NLS1 and NLS2 of pUL13 are functional NLS and that the synergistic effect of NLS1 and NLS2 can enhance their nuclear localization abilities. Studies on the DEV pUL13 homologous proteins HCMV UL97 and HHV U69 indicate that they facilitate nuclear transport through classical importin $\alpha$ and $\beta$ proteindependent pathways (Isegawa et al., 2008). It has been reported that ivermectin can block the interaction between importin $\alpha$ and importin $\beta$ to inhibit classical NLS-dependent nuclear import. However, ivermectin is a specific inhibitor of importin $\alpha$ - and $\beta$-mediated nuclear import, which inhibits replication of HIV-1 and dengue virus. Ivermectin can selectively inhibit NLS-mediated importin $\alpha$ - and importin $\beta$-dependent nuclear import and has no inhibitory effect on other nuclear import pathways (Wagstaff et al., 2012). Analysis of DEV pUL13 showed that the 2 NLS, NLS1 and NLS2, are classical 4- and 7-type NLS. To explore whether the entry of DEV pUL13 into the nucleus also occurs via a classical importin $\alpha$ - and $\beta$-dependent pathway, we treated cells with UL13-GFP and NLS1\&NLS2-GFP recombinant plasmids with ivermectin and compared fluorescence distribution between the treated group and the untreated group. The results showed that the distribution of fluorescence in the nucleus of the ivermectin-treated transfected cells was lower than that in the untreated cells, and the difference between the treated and untreated cells was very obvious. Ivermectin effectively inhibited the nuclear import of the DEV pUL13 and NLS1\&NLS2-GFP proteins, which indicated that the NLS of DEV pUL13 regulate nuclear import by forming a complex with importin $\alpha$ and $\beta$. It has been reported that ivermectin can block the interaction between importin $\alpha$ and importin $\beta$ to inhibit the classical NLS-dependent nuclear import (Wagstaff et al., 2012).

The cell biological characteristics of the successfully constructed NLS-deleted and NLS-rescued DEV CHv BAC UL13 $-\Delta$ NLS and $\Delta$ NLSR mutants were analyzed. There were no significant differences in plaque size, growth, or replication ability of cells infected with the UL13- $\Delta$ NLS recombinant virus and those of cells infected with the parent virus. The results showed that the pUL13 NLS had little effect on viral replication. Among the members of the $\beta$-herpesvirus subfamily and $\gamma$-herpesvirus subfamily, UL13 homologs have been reported to affect viral replication; for example, HCMV UL97 protein kinase affects viral replication by phosphorylating viral polymerase extension factor UL44 (Silva et al., 2011). EBV BGLF4 affects viral growth and replication by phosphorylating the viral elongation factor BMRF1 (Chen et al., 2000). And there are few reports on the effect of the UL13 protein on viral replication in members of the $\alpha$-herpesvirus subfamily. This may be related to the existence of another conserved protein kinase, US3 protein kinase, in the alpha herpesvirus subfamily. It has been reported that US3 protein kinase is an important but not essential gene on viral growth and replication (Reynolds et al., 2002). In the future studies, we can detect the effect of the UL13 NLS on the activity of pUL13 kinase and the biological function of DEV UL13 protein in the nucleus.

In conclusion, we determined that the nuclear import of DEV pUL13 is directed by aas 4 to 7 and 90 to 96 and proved that this nuclear import occurs via a classical importin $\alpha$ - and $\beta$-dependent process. We also constructed a mutant virus in which the NLS of $U L 13$ was deleted and preliminarily explored the characteristics of this mutant virus for further functional study.

\section{ACKNOWLEDGMENTS}

This work was supported by the National Key Research and Development Program of China (2017YFD0500800), the China Agricultural Research System (CARS-42-17), the Sichuan Veterinary Medicine and Drug Innovation Group of China Agricultural Research System (SCCXTD-2020-18), and Integration and Demonstration of Key Technologies for Goose Industrial Chain in Sichuan Province (2018NZ0005). The funders supervised the study but did not have any role in the collection, analysis, and interpretation of data or in writing the article.

\section{DISCLOSURES}

The authors declare no conflicts of interest.

\section{SUPPLEMENTARY DATA}

Supplementary data associated with this article can be found in the online version at https://doi.org/10.1 016/j.psj.2020.09.069.

\section{REFERENCES}

Akihisa, K., M. Yamamoto, T. Ohno, M. Tanaka, T. Sata, Y. Nishiyamaet, and Y. Kawaguchi. 2006. Herpes simplex virus 1encoded protein kinase UL13 phosphorylates viral Us3 protein kinase and regulates nuclear localization of viral envelopment factors UL34 and UL31. J. Virol. 80:1476-1486.

Baek, M. C., P. M. Krosky, A. Pearson, and D. M. Coen. 2004 Phosphorylation of the RNA polymerase II carboxyl-terminal domain in human cytomegalovirus-infected cells and in vitro by the viral UL97 protein kinase. Virology 324:184-193.

Cano-Monreal, G. L., K. M. Wylie, F. Cao, J. E. Tavis, and L. A. Morrison. 2009. Herpes simplex virus 2 UL13 protein kinas disrupts nuclear lamins. Virology 392:137-147. 
Chang, H., A. Cheng, M. Wang, Y. Guo, W. Xie, and Q. Luo. 2009. Complete nucleotide sequence of the duck plague virus $\mathrm{gE}$ gene. Arch. Virol. 154:163-165.

Chang, H., A. Cheng, M. Wang, D. Zhu, R. Jia, F. Liu, Z. Chen, Q. Luo, X. Chen, and Y. Zhou. 2010. Cloning, expression and characterization of $\mathrm{gE}$ protein of duck plague virus. Virol. J. 7:120.

Chang, Y., C. P. Lee, M. Su, J. Wang, J. Chen, S. Lin, C. Tsai, M. Hsieh, K. Takada, and M. Chenet. 2012. Epstein-Barr virus BGLF4 kinase retards cellular S-phase progression and induces chromosomal abnormality. PLoS One 7:e39217.

Chen, M., S. Chang, H. Huang, and J. Chen. 2000. A protein kinase activity associated with Epstein-Barr virus BGLF4 phosphorylates the viral early antigen EA-D in vitro. J. Virol. 74:3093-3104.

Cheng, A. 2015. Duck Plague. China Agriculture Press, Beijing.

Daikoku, T., S. Shibata, F. Goshima, S. Oshima, T. Tsurumi, H. Yamada, Y. Yamashita, and Y. Nishiyamaet. 1997. Purification and characterization of the protein kinase encoded by the UL13 gene of herpes simplex virus type 2. Enzyme Microb. Technol. 235:82-93.

Dhama, K., N. Kumar, M. Saminathan, R. Tiwari, K. Karthik, M. A. Kumar, M. Palanivelu, M. Z. Shabbir, Y. S. Malik, and R. K. Singh. 2017. Duck virus enteritis (duck plague) - a comprehensive update. Vet. Q. 37:57-80.

Gershburg, E., M. Marschall, K. Hong, and J. S. Pagano. 2004. Expression and localization of the Epstein-Barr virus-encoded protein kinase. J. Virol. 78:12140-12146.

Gershburg, S., L. Murphy, M. Marschall, and E. Gershburg. 2010. Key motifs in EBV (Epstein-Barr virus)-encoded protein kinase for phosphorylation activity and nuclear localization. Biochem. J. 431:227-235.

Guo, Y., C. Shen, A. Cheng, M. Wang, S. Chen, N. Zhang, and Y. Zhou. 2009. Anatid herpesvirus 1 virulent strain induces syncytium and apoptosis in duck embryo fibroblast cultures. Vet. Microbiol. 138:258-265.

Hu, X., M. Wang, S. Chen, R. Jia, D. Zhu, M. Liu, Q. Yang, K. Sun, X. Chen, and A. Cheng. 2017. The duck enteritis virus early protein, UL13, found in both nucleus and cytoplasm, influences viral replication in cell culture. Poult. Sci. 96:2899-2907.

Hwang, S., K. S. Kim, E. Flano, T. Wu, L. M. Tong, A. N. Park, M. J. Song, D. J. Sanchez, R. M. O'Connell, G. Cheng, and R. Sunet. 2009. Conserved herpesviral kinase promotes viral persistence by inhibiting the IRF-3-mediated type I interferon response. Cell Host Microbe 5:166-178.

Isegawa, Y. M., Y. Yasuda, K. Semi, K. Tsujimura, R. Fukunaga, A. Ohshima, A. Ohshima, Y. Horiguchi, Y. Yoneda, and N. Sugimoto. 2008. Characterization of the human herpesvirus 6 U69 gene product and identification of its nuclear localization signal. J. Virol. 82:710-718.

Kabachinski, G., and T. U. Schwartz. 2015. The nuclear pore complex-structure and function at a glance. J. Cell Sci. 128:423-429.

Krosky, P., M. C. Baek, and D. M. Coen. 2003. The human cytomegalovirus UL97 protein kinase, an antiviral drug target, is required at the stage of nuclear egress. J. Virol. 77:905-914.

Kudo, N., B. Wolff, T. Sekimoto, E. P. Schreiner, Y. Yoneda, M. Yanagida, S. Horinouchi, and M. Yoshidaet. 1998. Leptomycin $\mathrm{B}$ inhibition of signal-mediated nuclear export by direct binding to CRM1. Exp. Cell Res. 242:540-547.

Lee, C. P., Y. Huang, S. Lin, Y. Chang, Y. Chang, K. Takada, and M. Chen. 2008. Epstein-Barr virus BGLF4 kinase induces disassembly of the nuclear lamina to facilitate virion production. J. Virol. 82:11913-11926.

Leen, D., D. M. Bolle, T. Mertens, C. Manichanh, H. Agut, E. D. Clercq, E. De Clercq, and L. Naesens. 2002. Role of the human herpesvirus 6 U69-encoded kinase in the phosphorylation of ganciclovir. Mol. Pharmacol. 62:714-721.

Littler, E., A. D. Stuart, and M. S. Chee. 1992. Human cytomegalovirus UL97 open reading frame encodes a protein that phosphorylates the antiviral nucleoside analogue ganciclovir. Nature 358:160-162.

Liu, C., A. Cheng, M. Wang, S. Chen, R. Jia, D. Zhu, M. Liu, K. Sun, Q. Yang, and X. Chen. 2016. Characterization of nucleocytoplasmic shuttling and intracellular localization signals in duck enteritis virus UL54. Biochimie 127:86-94.

Ma, Y., Q. Zeng, M. Wang, A. Cheng, R. Jia, Q. Yang, Y. Wu, X. Zhao, M. Liu, D. Zhu, S. Chen, S. Zhang, Y. Liu, Y. Yu,
L. Zhang, and X. Chen. 2018. US10 protein is crucial but not indispensable for duck enteritis virus infection in vitro. Sci. Rep. 8:16510.

Marschall, M., M. Freitag, P. Suchy, D. Romaker, R. Kupfer, M. Hanke, and T. Stamminger. 2003. The protein kinase pUL97 of human cytomegalovirus interacts with and phosphorylates the DNA polymerase processivity factor pUL44. Virology 311:60-71.

Marschall, M., A. Marzi, P. aus dem Siepen, R. Jochmann, M. Kalmer, S. Auerochs, Lischka, Peter, Leis, Martina, and T. Stamminger. 2005. Cellular p32 recruits cytomegalovirus kinase pUL97 to redistribute the nuclear lamina. J. Biol. Chem. 280:33357-33367.

Michel, D., P. Schaarschmidt, K. Wunderlich, M. Heuschmid, L. Simoncini, D. Mühlberger, A. Zimmermann, I. Pavić, and T. Mertens. 1998. Functional regions of the human cytomegalovirus protein pUL97 involved in nuclear localization and phosphorylation of ganciclovir and pUL97 itself. J. Gen. Virol. 79:2105-2112.

Miller, M., M. K. Park, and J. A. Hanover. 1991. Nuclear pore complex: structure, function, and regulation. Physiol. Rev. 71:909-949.

Nakai, K., and P. Horton. 1999. PSORT: a program for detecting sorting signals in proteins and predicting their subcellular localization. Trends Biochem. Sci. 24:34-36.

Nishi, K., M. Yoshida, D. Fujiwara, M. Nishikawa, S. Horinouchi, and T. Beppu. 1994. Leptomycin B targets a regulatory cascade of crm1, a fission yeast nuclear protein, involved in control of higher order chromosome structure and gene expression. J. Biol. Chem. 269:6320-6324.

Ohshima, K., S. Takeda, M. Hirose, Y. Akiyama, K. Iguchi, M. Hoshino, K. Yamaguchi, and T. Mochizuki. 2012. Structure -function relationship of the nuclear localization signal sequence of parathyroid hormone-related protein. Biomed. Res. 33:191-199.

Paladino, P., E. Marcon, J. Greenblatt, and L. Frappier. 2014. Identification of herpesvirus proteins that contribute to G1/S arrest. J. Virol. 88:4480-4492.

Prichard, M. N., W. J. Britt, S. L. Daily, C. B. Hartline, and E. R. Kern. 2005. Human cytomegalovirus UL97 kinase is required for the normal intranuclear distribution of pp65 and virion morphogenesis. J. Virol. 79:15494-15502.

Reynolds, A. E., E. G. Wills, R. J. Roller, B. J. Ryckman, and J. D. Baines. 2002. Ultrastructural localization of the herpes simplex virus type 1 UL31, UL34, and US3 proteins suggests specific roles in primary envelopment and egress of nucleocapsids. J. Virol. 76:8939-8952.

Schmidt-Zachmann, M. S., and E. A. Nigg. 1993. Protein localization to the nucleolus:a search for targeting domains in nucleolin. J. Cell Sci. 105:799-806.

Shen, A., G. Ma, A. Cheng, M. Wang, D. Luo, L. Lu, T. Zhou, D. Zhu, Q. Luo, R. Jia, Z. Chen, Y. Zhou, and X. Chen. 2010. Transcription phase, protein characteristics of DEV UL45 and prokaryotic expression, antibody preparation of the UL45 des-transmembrane domain. Virol. J. 7:232.

Silva, L. A., B. L. Strang, E. W. Lin, J. P. Kamil, and D. M. Coen. 2011. Sites and roles of phosphorylation of the human cytomegalovirus DNA polymerase subunit UL44. Virology 417:268-280.

Tamrakar, S., A. J. Kapasi, and D. H. Spector. 2005. Human cytomegalovirus infection induces specific hyperphosphorylation of the carboxyl-terminal domain of the large subunit of RNA polymerase II that is associated with changes in the abundance, activity, and localization of cdk9 and cdk7. J. Virol. 79:15477-15493.

Tarakanova, V. L., V. Leung-Pineda, S. Hwang, C. Yang, K. Matatall, M. Basson, R. Sun, H. Piwnica-Worms, B. P. Sleckman, and H.W. Virgin, 4th. 2007. $\gamma$-Herpesvirus kinase actively initiates a DNA damage response by inducing phosphorylation of H2AX to foster viral replication. Cell Host Microbe 1:275-286.

Jacob, T., C. Van den Broeke, and H. W. Favoreel. 2011. Viral serine/ threonine protein kinases. J. Virol. 85:1158-1173.

Wagstaff, K. M., H. Sivakumaran, S. M. Heaton, D. Harrich, and D. A. Jans. 2012. Ivermectin is a specific inhibitor of importin alpha/beta-mediated nuclear import able to inhibit replication of HIV-1 and dengue virus. Biochem. J. 443:851-856.

Wang, J., S. L. Doong, S. C. Teng, C. P. Lee, C. H. Tsai, and M. R. Chen. 2009. Epstein-Barr virus BGLF4 kinase suppresses the interferon regulatory factor 3 signaling pathway. J. Virol. 83:18561869. 
Wu, Y., A. Cheng, M. Wang, Q. Yang, D. Zhu, R. Jia, S. Chen, Y. Zhou, and X. Chen. 2012. Complete genomic sequence of Chinese virulent duck enteritis virus. J. Virol. 86:5965.

Yang, P., S. Chang, C. Tsai, Y. Chao, and M. Chen. 2008. Effect of phosphorylation on the transactivation activity of Epstein-Barr virus BMRF1, a major target of the viral BGLF4 kinase. J. Gen. Virol. 89:884-895.

Yang, L., M. Wang, A. Cheng, Q. Yang, Y. Wu, R. Jia, M. Liu, D. Zhu, S. Chen, S. Zhang, X. Zhao, J. Huang, Y. Wang, Z. Xu, Z. Chen, L. Zhu, Q. Luo, Y. Liu, Y. Yu, L. Zhang, B. Tian, L. Pan, M. U. Rehman, and X. Chen. 2019. Innate immune evasion of alphaherpersvirus tegument proteins. Front. Immunol. 10:2196.

Yang, L., M. Wang, C. Zeng, Y. Shi, A. Cheng, M. Liu, D. Zhu, S. Chen, R. Jia, Q. Yang, Y. Wu, S. Zhang, X. Zhao, J. Huang, Y. Liu, X. O, S. Mao, Y. Yu, L. Zhang, B. Tian, L. Pan, M. U. Rehman, and X. Chen. 2020. Duck enteritis virus UL21 is a late gene and encodes a protein that interacts with pUL16. BMC. Vet. Res. 16:8.

Yokota, S., N. Yokosawa, T. Okabayashi, T. Suzutani, S. Miura, K. Jimbow, and N. Fujii. 2004. Induction of suppressor of cytokine signaling- 3 by herpes simplex virus type 1 contributes to inhibition of the interferon signaling pathway. J. Virol. 78:6282-6286.
You, Y., T. Liu, M. Wang, A. Cheng, R. Jia, Q. Yang, Y. Wu, D. Zhu, S. Chen, M. Liu, X. Zhao, S. Zhang, Y. Liu, Y. Yu, and L. Zhang. 2018. Duck plague virus glycoprotein J is functional but slightly impaired in viral replication and cell-to-cell spread. Sci. Rep. 8:4069.

Zhang, S., J. Xiang, A. Cheng, M. Wang, X. Li, L. Li, X. Chen, D. Zhu, Q. Luo, and X. Chen. 2010. Production, purification and characterization of polyclonal antibody against the truncated gK of the duck enteritis virus. Virol. J. 7:241.

Zhao, L., A. Cheng, M. Wang, G. Yuan, and M. Cai. 2008. Characterization of codon usage bias in the d UTPase gene of duck enteritis virus. Prog. Nat. Sci. 18:1069-1076.

Zhao, C., T. He, Y. Xu, M. Wang, A. Cheng, X. Zhao, D. Zhu, S. Chen, M. Liu, Q. Yang, R. Jia, X. Chen, Y. Wu, S. Zhang, Y. Liu, Y. Yu, and L. Zhang. 2019. Molecular characterization and antiapoptotic function analysis of the duck plague virus Us5 gene. Sci. Rep. $9: 4851$.

Zhou, X., M. Wang, A. Cheng, Q. Yang, Y. Wu, R. Jia, M. Liu, D. Zhu, S. Chen, S. Zhang, X. Zhao, J. Huang, S. Mao, X. Ou, Q. Gao, Y. Liu, Y. Yu, L. Zhang, B. Tian, L. Pan, M. U. Rehman, and $\mathrm{X}$. Chen. 2020. Development a simple and rapid immunochromatographic strip test for detecting antibody of duck plague virus based on gI protein. J. Virol. Method 277:113803. 